\title{
Subunit-Specific Regulation of NMDA Receptor Endocytosis
}

\author{
Gabriela Lavezzari, Jennifer McCallum, Colleen M. Dewey, and Katherine W. Roche \\ National Institute of Neurological Disorders and Stroke, National Institutes of Health, Bethesda, Maryland 20892
}

At excitatory synapses, both NMDA and AMPA receptors are localized to the postsynaptic density (PSD). However, unlike AMPA receptors, synaptic NMDA receptors are stable components of the PSD. Even so, surface-expressed NMDA receptors undergo endocytosis, which is more robust early in development and declines during synaptic development. We investigated the subunit-specific contributions to NMDA receptor endocytosis, specifically defining the endocytic motifs and endocytic pathways preferred by the NR2A and NR2B subunits. We find that NR2A and NR2B have distinct endocytic motifs encoded in their distal C termini and that these interact with clathrin adaptor complexes with differing affinities. We also find that NR2A and NR2B sort into different intracellular pathways after endocytosis, with NR2B preferentially trafficking through recycling endosomes. In mature cultures, we find that NR2B undergoes more robust endocytosis than NR2A, consistent with previous studies showing that NR2A is more highly expressed at stable synaptic sites. Our findings demonstrate fundamental differences between NR2A and NR2B that help clarify developmental changes in NMDA receptor trafficking and surface expression.

Key words: internalization; clathrin; endocytosis; glutamate receptors; trafficking; endocytic sorting

\section{Introduction}

Glutamate receptors mediate the majority of excitatory neurotransmission in the CNS and are essential for synaptic development and plasticity. Although the synaptic localization of glutamate receptors is tightly regulated, it is becoming clear that the targeting and anchoring of the various subtypes of glutamate receptors are differentially regulated. For example, synaptic AMPA receptors undergo rapid and robust cycling at the postsynaptic membrane (Luscher et al., 1999; Sheng, 2001; Malinow and Malenka, 2002), whereas synaptic NMDA receptors are relatively stable (Luscher et al., 1999; Wenthold et al., 2003). Although synaptic NMDA receptors are tightly anchored to the postsynaptic density (PSD), it is clear that NMDA receptors are quite mobile within neurons, via intracellular pathways and lateral diffusion in the membrane (Rao and Craig, 1997; Tovar and Westbrook, 1999; Standley et al., 2000; Scott et al., 2001; Snyder et al., 2001; Xia et al., 2001; Washbourne et al., 2002). In addition, surface-expressed NMDA receptors have been shown to internalize both in primary neuronal cultures and when expressed in heterologous cells (Roche et al., 2001). The regulation of NMDA receptor endocytosis is therefore likely to be a critical determinant of NMDA receptor surface expression, both at synaptic and extrasynaptic sites.

NMDA receptors are tetramers comprising NR1, NR2 (NR2A-NR2D), and NR3 (NR3A-NR3B) subunits. In the forebrain, endogenous NMDA receptors are thought to be composed

Received Feb. 6, 2004; accepted May 28, 2004

We thank Dayna Hayes for contribution to the preparation of the primary neuronal cultures, the National Institute of Neurological Disorders and Stroke (NINDS) Light Imaging Facility, in particular the facility manager Dr. Carolyn Smith, for advice and expertise in the collection and analysis of the confocal images, and the NINDS Sequencing Facility for automated DNA sequence analysis.

Correspondence should be addressed to K. W. Roche, National Institute of Neurological Disorders and Stroke, National Institutes of Health, Building 36, Room 5B20, Bethesda, MD 20892. E-mail: rochek@ninds.nih.gov. DOI:10.1523/JNEUROSCI.1890-04.2004

Copyright $\odot 2004$ Society for Neuroscience $\quad$ 0270-6474/04/246383-09\$15.00/0 of NR1 subunits combined with NR2B early in development and heteromeric combinations of NR1, NR2B, and NR2A in mature neurons (Kew et al., 1998; Li et al., 1998; Vicini et al., 1998; Rumbaugh and Vicini, 1999; Tovar and Westbrook, 1999). NMDA receptor endocytosis is developmentally regulated, with robust endocytosis early in development that declines as neurons mature and synapses form (Roche et al., 2001). Interestingly, the decline in NMDA receptor endocytosis correlates well with the known changes in NMDA receptor subunit composition during development. Previous studies have defined an endocytic motif encoded within the NR2B subunit. This motif, YEKL, is located just upstream of the PSD-95-Discs large-zona occludens-1 (PDZ)-binding domain on the extreme $\mathrm{C}$ terminus of NR2B (Roche et al., 2001). However, it is not known whether NR2A also contains endocytic motifs within its $\mathrm{C}$ terminus.

In the current study, we have characterized the specific contributions of the NR2B and NR2A subunits to NMDA receptor endocytosis using a variety of approaches. We find that NR2A and NR2B contain different sorting motifs within their distal C termini that regulate trafficking to distinct intracellular pathways after endocytosis. In neurons, we find that NR2B preferentially sorts to recycling endosomes, consistent with the more robust internalization of NR2B-containing NMDA receptors. NR2B expression drives NMDA receptor endocytosis in both immature and mature neurons, whereas NR2A endocytosis is most notable at early stages of development. These results are consistent with the presence of stable NR2A-containing NMDA receptor complexes at mature synapses. Our findings demonstrate a strong subunit-specific contribution to NMDA receptor endocytosis and stability on the plasma membrane.

\section{Materials and Methods}

Antibodies and DNA constructs. The primary antibodies Tac 7G7 (American Type Culture Collection, Manassas, VA) and FLAG M2 (Sigma, St. 
Louis, MO) were used for immunofluorescence. All secondary antibodies were obtained from Molecular Probes (Eugene, OR), except for cyanine 5 (Cy5)-conjugated anti-rabbit, which was obtained from Jackson ImmunoResearch (West Grove, PA). The following cDNA constructs were obtained as gifts: FLAG-NR2B (F. A. Stephenson, School of Pharmacy, University of London, London, UK), green fluorescent protein (GFP)-Rab5, GFP-Rab7, GFP-Rab9, $\mu 1, \mu 2, \mu 3 \mathrm{a}$, and $\mu 4$ in pACTII, and the trans-Golgi network (TGN) 38 full C terminus in pGBT9 (Juan Bonifacino, National Institute of Child Health and Human Development, National Institutes of Health, Bethesda, MD); and GFP-Rab11 (James Goldenring, Vanderbilt University, Nashville, TN). The TGN38 $\mathrm{C}$ terminus was subcloned into the pBHA vector using EcoRI and PstI restriction sites. Both the NR2A and NR2B C termini were amplified by PCR. The amplified tails included amino acids 1315-1482 (NR2B) or 1304-1464 (NR2A) and were subcloned using EcoRI restriction sites into pBHA vector or with $E c o$ RV and $X b a I$ into Tac vector. The TacNR2A truncations, TacNR2A $\Delta 7$ and TacNR2A $\Delta 11$, were obtained using PCR. FLAG-NR2A was constructed in the laboratory using PCR with the following sequence: 5'-GAACTTCGAAATCTGGACTACAAGGACGACGATGACAAGTGGGGCCCAGAGCAG-3' and 3'-CTGCTCTGGGCCCCACTTGTCATCGTCGTCCTTGTAGTCCAGATTTCGAAGTTC-5', inserting the FLAG epitope between amino acids 54 and 55 of NR2A. The bold sequence indicates the FLAG peptide. FLAG-NR2A $\Delta 11$ L1320A was constructed in the laboratory using site-directed mutagenesis. All mutations were confirmed using automated sequence analysis.

Yeast two-hybrid direct-interaction assays. Direct-interaction assays of NR2B (amino acids 1315-1482), NR2A (1304-1464), or the TGN38 C terminus binding to adaptor medium chains were performed as described previously (Kim et al., 1995). Briefly, NR2B (amino acids 13151482), NR2A (1304-1464), or TGN38 in the LexA DNA binding domain vector pBHA and medium chains in the GAL4 activation domain vector pACTII were cotransformed into L40 yeast cells. For each condition, one-half of the yeast cells were plated on dextrose plates containing histidine but lacking leucine and tryptophan (His-containing), and the other half were plated on dextrose plates lacking histidine, leucine, and tryptophan (His-deficient). Nutritional selection was used as a measure of a direct interaction. For nutritional selection, growth on His-deficient plates was scored on a - to ++++ scale for conditions with equivalent growth on His-containing plates.

Immunofluorescent internalization assay in HeLa cells. HeLa cells (American Type Culture Collection) grown on coverslips were transfected with the cDNAs indicated ( $4 \mu \mathrm{g}$ of DNA per well in a six well dish) using the calcium phosphate coprecipitation method, and experiments were performed $48 \mathrm{hr}$ later. Transfected cells were washed in PBS, incubated with anti-Tac antibody for $1 \mathrm{hr}$ on ice, washed with PBS, and returned to conditioned media at $37^{\circ} \mathrm{C}$ for 15 min to allow internalization. The cells were washed in PBS, fixed in 4\% paraformaldehyde in PBS for $15 \mathrm{~min}$, washed in PBS, and permeabilized in $0.25 \%$ Triton X-100 in PBS for $5 \mathrm{~min}$. The coverslips were washed in PBS, incubated with Alexa 568-conjugated anti-mouse secondary antibodies for $30 \mathrm{~min}$ at room temperature, washed, and mounted with ProLong Antifade Kit (Molecular Probes). When double labeling [early endosome autoantigen 1 (EEA1); PSD-95], the cells were incubated with the appropriate primary antibody for $1 \mathrm{hr}$ at room temperature after permeabilization. The cells were washed in PBS, incubated with both anti-mouse and anti-rabbit secondary antibodies (1:500) for $30 \mathrm{~min}$ at room temperature, washed in PBS, and mounted. Images were collected with a $63 \times$ objective on a Zeiss (Oberkochen, Germany) LSM 510. Series of optical sections were collected at intervals of $0.34 \mu \mathrm{m}$. Figures show maximum projections. For the statistical analysis, images from five different HeLa cells in three independent experiments were collected at a $63 \times$ objective with a Zeiss Axioplan 2 microscope and analyzed with the OpenLab colocalization module (Improvision, Lexington, MA). Values represent the mean \pm SEM of Pearson's correlation.

Quantitative assay of internalization in HeLa cells. HeLa cells were grown on 12 well dishes and transiently transfected with Tac-NMDA receptor chimeras. The cells were incubated with anti-Tac antibody for 1 hr on ice to label surface-expressed protein. Cells were either left on ice or washed and returned to conditioned media for $15 \mathrm{~min}$ at $37^{\circ} \mathrm{C}$ to allow internalization. Cells were then fixed for $15 \mathrm{~min}$ in 4\% paraformaldehyde without detergent permeabilization. After fixation, the cells were washed and incubated for $45 \mathrm{~min}$ with [ $\left.{ }^{125} \mathrm{I}\right]$-labeled anti-mouse antibody $(25$ $\mu \mathrm{Ci})$ at room temperature. After extensive washing, the cells were incubated with $1 \mathrm{M} \mathrm{NaOH}$ for $15 \mathrm{~min}$ at room temperature, and the lysate was collected and analyzed. Each condition was assayed in triplicate. For each experiment, background antibody binding was measured on cells transfected with empty vector (also in triplicate), and the average background value was subtracted from the average experimental counts. Quantitative assessment of total surface labeling was calculated by measuring the amount of anti-Tac antibody present on cells maintained on ice and not subjected to the $15 \mathrm{~min}, 37^{\circ} \mathrm{C}$ incubation. Quantitative assessment of internalization was calculated by subtracting the amount of anti-Tac antibody remaining on cells after the $15 \mathrm{~min}, 37^{\circ} \mathrm{C}$ incubation from the total surface counts. Results are expressed as a ratio of internalization to total. The graph in Figure $2 B$ represents the average of five independent experiments.

Transfected hippocampal neurons. Preparation of primary hippocampal cultures was performed as described previously (Roche and Huganir, 1995) with minor changes. Briefly, cells were dissected from embryonic day 18 Sprague Dawley rats (Harlan, Indianapolis, IN), dissociated in $0.25 \%$ trypsin, and incubated with $0.01 \%$ DNase. Dispersed neurons were plated onto polyornithine-fibronectin-coated glass coverslips at a density of 100,000 cells per well of a 12 well dish. The neurons were grown in serum-free media (Neurobasal; Invitrogen, Gaithersburg, MD) with B27 supplement (Invitrogen). The cultures were maintained in a $5 \% \mathrm{CO}_{2}$ incubator until transfected and processed for immunofluorescence. Hippocampal neurons [ 3 or $10 \mathrm{~d}$ in vitro (DIV)] were transfected with the appropriate cDNA using the calcium phosphate coprecipitation method. Six micrograms of total DNA were used per well of the 12 well dish. Precipitation was allowed to form on cells for $\sim 45 \mathrm{~min}$, and the cells were washed twice with serum-free media. The cells were returned to conditioned media and maintained at $37^{\circ} \mathrm{C}$ in a $5 \% \mathrm{CO}_{2}$ incubator for an additional $48 \mathrm{hr}$. The use and care of animals used in this study followed the guidelines of the National Institutes of Health Animal Research Advisory Committee.

Internalization and immunocytochemistry of neurons. Transfected neurons were washed in PBS and incubated with polyclonal FLAG antibody for $45 \mathrm{~min}$ at room temperature to label surface-expressed protein. The antibody was removed, and the coverslips were returned to conditioned media for 15 or $30 \mathrm{~min}$ at $37^{\circ} \mathrm{C}$. The cells were washed in PBS and fixed with $4 \%$ paraformaldehyde- $4 \%$ sucrose in PBS for 15 min. The cells were incubated for $30 \mathrm{~min}$ at room temperature with Alexa 568conjugated (red) anti-rabbit secondary antibody (Molecular Probes) for labeling the surface population. The cells were washed and permeabilized in $0.25 \%$ Triton X-100 in PBS for 5 min. After a 30 min incubation with $10 \%$ normal goat serum (Vector Laboratories, Burlingame, CA), the cells were then incubated with Alexa 488-conjugated (green) anti-rabbit secondary antibody for $30 \mathrm{~min}$ to specifically label the internalized population. The cells were washed in PBS and mounted with a ProLong Antifade kit (Molecular Probes). When neurons were cotransfected with GFP constructs, the surface population was labeled with Alexa 568conjugated (red) anti-rabbit secondary antibody, and after permeabilization, the internalized population was specifically labeled with Cy5conjugated (blue) anti-rabbit secondary antibody from Jackson ImmunoResearch. Images were collected with a $63 \times$ objective on a Zeiss LSM 510. Series of optical sections were collected at intervals of $0.34 \mu \mathrm{m}$. Figures show maximum projections. For quantitative analysis, images from three dendrites per neuron (three neurons per experiment) were collected, and colocalization analysis was measured based on the data collected in three to five independent experiments with Volocity 2 software (Improvision). Values represent mean \pm SEM; $n=5$.

\section{Results}

\section{The NR2A C terminus contains an internalization motif}

Endogenous NMDA receptors undergo robust endocytosis that decreases as synapses develop and neurons mature. We have demonstrated previously that NR2B contains important regulatory motifs that influence NMDA receptor internalization and 
A

\section{NR2A C-10minus (1004-1464)}

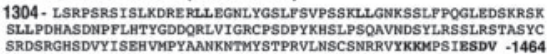

B

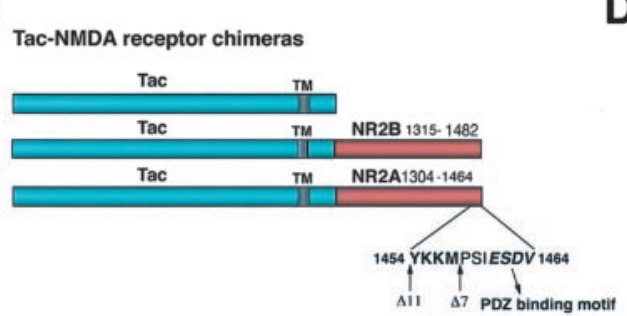

C
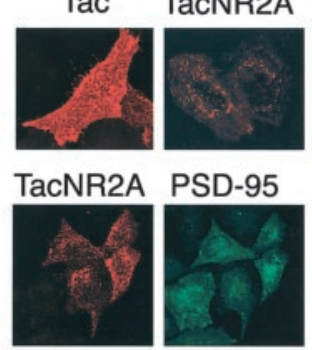

Figure 1. The C terminus of NR2A contains an endocytic motif that is inhibited by PSD-95 binding. $A$, The amino acid sequence of the distal C terminus of NR2A. Potential endocytic motifs (LL and YXXØ) and the PDZ ligand (ESDV) are indicated in bold. $B$, Schematic diagrams of the Tac-NMDA receptor chimeras. The distal C terminus of NR2B (amino acids 1315-1482) or NR2A (amino acids 1304-1464) was appended to the plasma membrane reporter molecule Tac (TacNR2B; TacNR2A). TacNR2A $\Delta 7$ (NR2A amino acids 1304-1457) is a truncation removing the PDZ-binding domain. TacNR2A 11 (amino acids 1304-1453) is a truncation removing both the PDZ-binding domain and the putative tyrosine-based endocytic motif. The transmembrane domain is depicted as a striped rectangle, and the putative tyrosine-based motif and the PDZ-binding domain are depicted in bold. C, The NR2B and NR2A C termini are both sufficient to induce internalization of the surface protein Tac into endosomal structures. HeLa cells were transiently transfected with Tac, TacNR2B, or TacNR2A. Cells were incubated with Tac monoclonal antibody on ice for 1 $\mathrm{hr}$ and then returned to conditioned media and incubated at $37^{\circ} \mathrm{C}$ for $15 \mathrm{~min}$. The cells were fixed and permeabilized, and the immunoreactivity was visualized using Alexa 568-conjugated (red) anti-mouse secondary antibodies. Images were collected with a $63 \times$ objective on a Zeiss $L S M$. Series of optical sections were collected at intervals of $0.34 \mu \mathrm{m}$. Maximum projections are shown. D, PSD-95 inhibits internalization of TacNR2A and clusters the chimera on the cell surface. HeLa cells were transiently transfected with PSD-95 and TacNR2A. Cells were incubated with Tac monoclonal antibody on ice for $1 \mathrm{hr}$ and then returned to conditioned media and incubated at $37^{\circ} \mathrm{C}$ for $15 \mathrm{~min}$. The cells were fixed, permeabilized, and incubated with PSD-95 polyclonal antibodies. Immunoreactivity was visualized using a combination of Alexa 568-conjugated (red) anti-mouse and Alexa 488-conjugated (green) anti-rabbit secondary antibodies. Images were collected with a $63 \times$ objective on a Zeiss LSM. Series of optical sections were collected atintervals of $0.34 \mu \mathrm{m}$. Maximum projections are shown.

stability on the plasma membrane (Roche et al., 2001; Lavezzari et al., 2003). Whereas NR2B is expressed early in development, NR2A is only expressed in mature neurons. To determine whether this developmental change in NR2 subunit expression influences the surface stability of NMDA receptors, we have begun to identify sequences within NR2A that might regulate its surface expression. Like NR2B, NR2A contains a PDZ-binding domain motif at the extreme C terminus (-ESDV) (Fig. 1 $A, B$ ). In addition, NR2A contains several potential internalization motifs, including a putative tyrosine-based endocytic motif and several dileucine motifs. Both of these are consensus endocytic motifs that can regulate clathrin-mediated endocytosis (Ohno et al., 1995, 1996; Bonifacino and Traub, 2003). It is known that NR2B contains a tyrosine-based endocytic motif (YEKL) that binds directly to the AP-2 adaptor complex (Lavezzari et al., 2003) and regulates endocytosis. Although the tyrosine residue is conserved in both NR2A and NR2B, the surrounding residues in each subunit are unique (YKKM vs YEKL) (Fig. $1 A, B$ ).

To determine whether any of these putative endocytic motifs within the NR2A C terminus regulate surface expression, we have created a chimera of the distal portion of NR2A (amino acids 1304-1464) and the stable surface integral membrane protein Tac (TacNR2A) (Fig. 1B), and evaluated endocytosis of this chimera compared with wild-type Tac and the TacNR2B chimera (NR2B amino acids 1315-1482) (Fig. 1B). We were unable to compare the distal $\mathrm{C}$ terminus to the entire $\mathrm{C}$ terminus, because Tac chimeras using the entire $C$ termini of NR2A or NR2B are retained by the endoplasmic reticulum (ER) (data not shown) (Hawkins et al., 2004). Transfected HeLa cells were surfacelabeled on ice for $1 \mathrm{hr}$ with Tac antibody and returned to conditioned media for 15 min to allow internalization. Whereas Tac alone was expressed almost exclusively on the plasma membrane, TacNR2A was internalized into vesicular structures similar to TacNR2B (Fig. 1C). These data show that the $\mathrm{C}$ terminus of NR2A contains a sequence that is sufficient to induce internalization of Tac. Within the NR2A C terminus there is also the PDZ domainbinding motif ( - ESDV) that is completely conserved with NR2B and binds to the PSD-95 family of proteins (Kim et al., 1995; Kornau et al., 1995; Sheng, 2001). To examine the role of PSD-95 in regulating surface expression, we coexpressed TacNR2A with PSD-95 in HeLa cells and evaluated internalization of TacNR2A. As we had observed previously in our examination of NR2B-mediated endocytosis (Roche et al., 2001; Lavezzari et al., 2003), coexpression of PSD-95 blocked the internalization of TacNR2A and clustered TacNR2A into patches on the cell surface (Fig. 1D). Coexpression of PSD-95 with TacNR2A $\Delta 7$, which does not contain the PDZ-binding motif, did not affect internalization of the chimera (data not shown), demonstrating that the direct interaction with PSD-95 is essential.

To determine whether the conserved tyrosine within the YXX $\varnothing$ endocytic motif in $\mathrm{NR} 2 \mathrm{~A}$ regulates endocytosis, we truncated the last 11 amino acids of the TacNR2A C terminus to remove this putative motif (TacNR2A $\Delta 11$ ) (Fig. $1 B$ ). However, we found that this mutation did not suppress internalization (Fig. 2). This result is in sharp contrast to previous findings demonstrating the importance of the analogous tyrosine (1472) on the endocytosis of TacNR2B (Roche et al., 2001; Lavezzari et al., 2003). This result led us to investigate whether the dileucine motifs in the NR2A C terminus were critical for internalization. We used site-directed mutagenesis and mutated the three dileucine motifs from LL to LA (TacNR2A $\Delta 11$ L1320A; TacNR2A $\Delta 11$ L1337A; TacNR2A $\Delta 11$ L1358A). Both the immunofluorescence assay for internalization (Fig. 2A) and an independent quantitative measure of endocytosis (Fig. 2B) demonstrated that endocytosis of TacNR2A $\Delta 11$ L1320A was significantly less compared with the other TacNR2A chimeras. This therefore indicates that leucine 1320 regulates endocytosis of TacNR2A.

\section{Differential binding of NR2A and NR2B to adaptor medium chains}

The medium chain of AP-2, $\mu 2$, binds directly to internalized cargo proteins, thereby linking them to clathrin and regulating endocytosis (Ohno et al., 1995, 1996; Bonifacino and Traub, 2003). Although we have shown previously that the $C$ terminus of NR2B interacts directly with $\mu 2$ (Lavezzari et al., 2003), we wanted to determine whether the conserved tyrosine in the NR2A $\mathrm{C}$ terminus also binds to $\mu 2$. Using the yeast two-hybrid assay, we found that unlike NR2B, the NR2A C terminus did not interact with $\mu 2$ (Fig. 3). In addition to AP-2, there are other intracellular adaptor complexes (AP-1, AP-3, and AP-4), each of which possesses a unique medium chain and each of which regulates distinct transport steps in the endocytic pathway. Therefore, we examined the ability of NR2A and NR2B to interact with different 
A
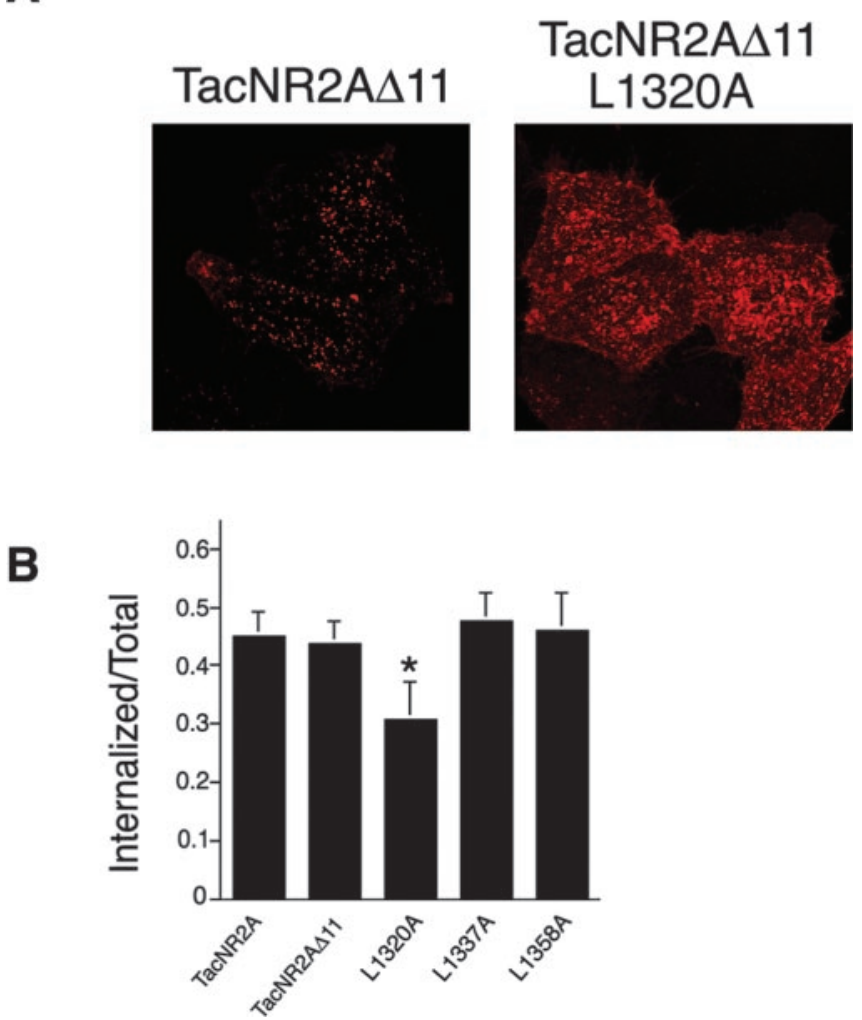

Figure 2. TacNR2A internalization is dependent on a dileucine motif. $A$, HeLa cells were transiently transfected with TacNR2A $\Delta 11$ or TacNR2A $\Delta 11$ L1320A. Cells were incubated with Tac monoclonal antibody on ice for $1 \mathrm{hr}$ and then returned to conditioned media and incubated at $37^{\circ} \mathrm{C}$ for $15 \mathrm{~min}$. The cells were fixed and permeabilized, and the immunoreactivity was visualized using Alexa 568-conjugated (red) anti-mouse secondary antibodies. Images were collected with a $63 \times$ objective on a Zeiss LSM. Series of optical sections were collected at intervals of $0.34 \mu \mathrm{m}$. Maximum projections are shown. $B$, HeLa cells were transiently transfected with TacNR2A, TacNR2A $\Delta 11$, TacNR2A $\Delta 11$ L1320A, TacNR2A $\Delta 11$ L1337A, or TacNR2A $\Delta 11$ L1358A. The amount of internalization was determined using an independent quantitative internalization assay as described in Materials and Methods. Values are expressed as internalization per total (the total represents initial surface labeling; mean $\pm \mathrm{SEM} ; n=5$ ). Statistical comparison was performed using ANOVA. ${ }^{*} p<0.01$.

adaptor medium chains in the yeast two-hybrid assay. The well characterized binding between the adaptor medium chains and the TGN38 C terminus was used as a positive control (Aguilar et al., 2001). Most notable were the interactions with $\mu 1$ and $\mu 2$. Interestingly, we found that the NR2A and NR2B C termini bind equally well to $\mu 1$, the adaptor subunit that plays a role in clathrin vesicle formation at the TGN. However, the plasma membrane adaptor subunit $\mu 2$ interacted robustly with NR2B but not NR2A. This is consistent with the YKKM motif in NR2A not playing a critical role in plasma membrane endocytosis but potentially being important for other intracellular sorting steps. This correlates quite well with results of our endocytosis assays (Fig. 2), which also revealed that the YKKM motif does not play an important role in endocytosis.

\section{The NR2A and NR2B C termini regulate trafficking to distinct} intracellular pathways

After we determined that the $\mathrm{C}$ terminus of NR2A was sufficient to induce endocytosis of the stable surface protein Tac, we began to characterize the intracellular compartments that contained internalized TacNR2A. Previous data demonstrated that TacNR2B is colocalized with internalized transferrin (Roche et al., 2001),

\begin{tabular}{cccc}
$\begin{array}{c}\text { Adaptor } \\
\text { medium chain }\end{array}$ & $\begin{array}{c}\text { NR2A C-terminus } \\
\text { (a.a. 1304-1464) } \\
\text { His 3 }\end{array}$ & $\begin{array}{c}\text { NR2B C-terminus } \\
\text { (a.a. 1315-1482) } \\
\text { His 3 }\end{array}$ & $\begin{array}{c}\text { TGN38 } \\
\text { C-terminus } \\
\text { His 3 }\end{array}$ \\
\hline$\mu 1$ & +++ & ++++ & ++++ \\
$\mu 2$ & + & +++ & ++++ \\
$\mu 3 \alpha$ & + & ++ & ++++ \\
$\mu 4$ & +++ & +++ & -
\end{tabular}

Figure 3. The NR2A C terminus and NR2B C terminus differentially bind to the medium chains of adaptor complexes. The NR2A C terminus (amino acids 1304-1464), the NR2B C terminus (amino acids 1315-1482), or the TGN38 C terminus in the LexA DNA binding domain was cotransformed with $\mu 1, \mu 2, \mu 3 \alpha$, or $\mu 4$ in the GAL 4 activation domain as follows: (1) $\mu 1$ and NR2A, (2) $\mu 2$ and NR2A, (3) $\mu 3 \alpha$ and NR2A, (4) $\mu 4$ and NR2A, (5) $\mu 1$ and NR2B, (6) $\mu 2$ and NR2B, (7) $\mu 3 \alpha$ and NR2B, (8) $\mu 4$ and NR2B, (9) $\mu 1$ and TGN38, (10) $\mu 2$ and TGN38, (11) $\mu 3 \alpha$ and TGN38, and (12) $\mu 4$ and TGN38. Growth on His-deficient plates was scored on a scale of - to ++++ for conditions with equivalent growth on His-containing plates.

revealing its presence in early endosomes. To determine whether TacNR2A follows the same intracellular trafficking pathway as TacNR2B, we followed the same approach as before, but observed that although TacNR2B and TacNR2A both traffic to early endosomes initially, they subsequently traffic to distinct compartments over time. To characterize the intracellular sorting, we analyzed TacNR2A and TacNR2B over time using immunofluorescence microscopy to examine their subcellular localization. We incubated the cells on ice for $1 \mathrm{hr}$ with Tac antibody and then returned the cells to $37^{\circ} \mathrm{C}$ for various times to allow internalization. After $5 \mathrm{~min}$, TacNR2A colocalized completely with the early endosome marker GFP-Rab5 (Fig. 4A). At $15 \mathrm{~min}$, we saw that the amount of colocalization with GFP-Rab5 decreased and TacNR2A trafficked into a different compartment (data not shown). To identify this compartment we coexpressed the late endosome marker GFP-Rab9 in these cells. At $15 \mathrm{~min}$, we were able to see partial colocalization of TacNR2A with GFP-Rab9positive late endosomes, and by $30 \mathrm{~min}$, we found almost complete colocalization (Fig. $4 B-D$ ).

We then quantitated the colocalization of TacNR2A with the early endosomal marker EEA1 and the late endosomal marker GFP-Rab9 over time (5, 15, and $30 \mathrm{~min}$ ) (Fig. $4 E$ ). At $5 \mathrm{~min}$, TacNR2A colocalized well with EEA1 but not well with GFPRab9. At $15 \mathrm{~min}$, there was moderate colocalization with both endosomal markers, and at $30 \mathrm{~min}$, TacNR2A strongly colocalized with GFP-Rab9, demonstrating that the NR2A C terminus regulates sorting from early to late endosomes.

We had originally observed strong colocalization of TacNR2B with transferrin at both 5 and $15 \mathrm{~min}$ (data not shown) (Roche et al., 2001). In contrast to TacNR2A, we found very little colocalization of TacNR2B with GFP-Rab9 after $30 \mathrm{~min}$, indicating that the NR2B C terminus did not regulate sorting to late endosomes. At this time point, most of the internalized TacNR2B was distributed throughout the cell, with some accumulation at the cell edges. This expression pattern was very different from the strong perinuclear distribution of GFP-Rab9 that colocalized with internalized TacNR2A after $30 \mathrm{~min}$. To define the compartment to which TacNR2B trafficked at later time points, we coexpressed cells with GFP-Rab11, a marker for recycling endosomes. After 30 min of internalization, we observed that the majority of internalized TacNR2B colocalized with GFP-Rab11 (Fig. 5A). These data are consistent with the idea that the NR2B C terminus reg- 
A
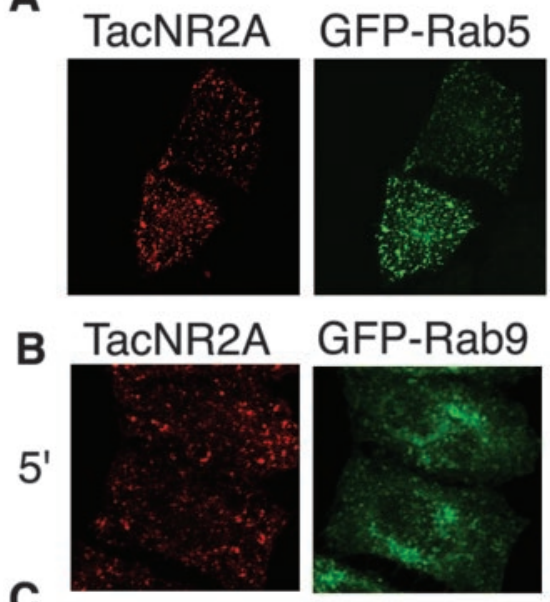

C

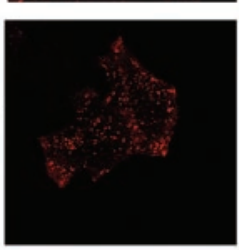

D
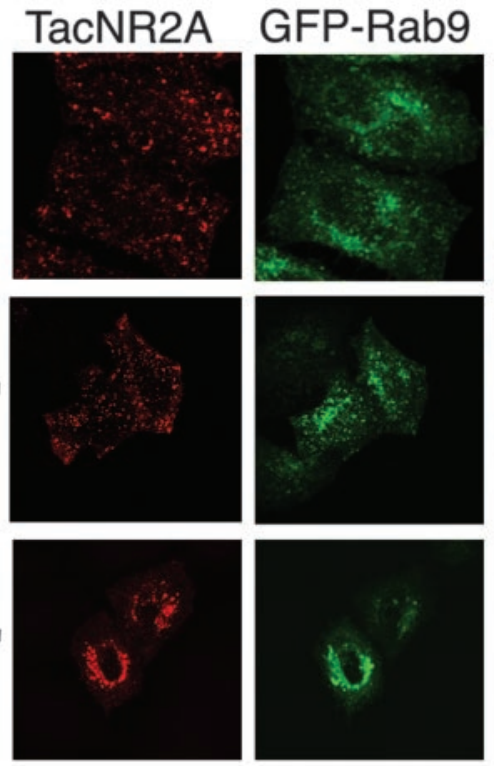

E

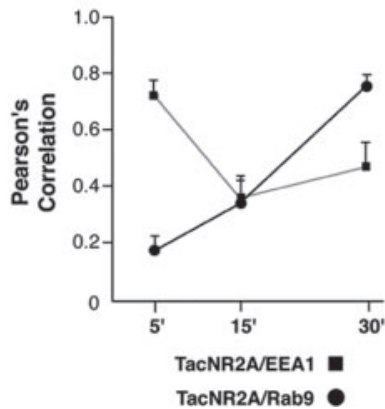

Figure 4. The NR2A C terminus regulates trafficking from early endosomes to late endosomes. A, After endocytosis, TacNR2A is localized to early endosomes at early time points. HeLa cells were transiently transfected with TacNR2A and GFP-Rab5. Cells were incubated with Tac polyclonal antibody on ice for $1 \mathrm{hr}$ and then returned to conditioned media for 5 min at $37^{\circ} \mathrm{C}$. The cells were fixed and permeabilized, and the immunoreactivity was visualized using Alexa 568-conjugated (red) antimouse. $B-D, 0$ ver time, TacNR2A traffics to late endosomes. HeLa cells were transiently transfected with TacNR2A and GFP-Rab9. Cells were incubated with Tac polyclonal antibody on ice for $1 \mathrm{hr}$ and then returned to conditioned media for $5 \mathrm{~min}(B), 15 \mathrm{~min}(C)$, or $30 \mathrm{~min}(D)$ at $37^{\circ} \mathrm{C}$. The cells were fixed and permeabilized, and the immunoreactivity was visualized using Alexa 568-conjugated (red) anti-mouse. Images were collected with a $63 \times$ objective on a Zeiss LSM. Series of optical sections were collected at intervals of $0.34 \mu \mathrm{m}$. Maximum projections are shown. $E$, Analysis of the amount of colocalization between TacNR2A and EEA1 or GFP-Rab9. HeLa cells were transiently transfected with TacNR2A. Cells were incubated with Tac polyclonal antibody on ice for $1 \mathrm{hr}$ and then returned to conditioned media for 5,15, or 30 min at $37^{\circ} \mathrm{C}$. The cells were fixed, permeabilized, and incubated with antibody for early endosomal marker EEA1. Immunoreactivity was visualized using a combination of Alexa 568-conjugated (red) anti-mouse lgG2A and Alexa 488-conjugated (green) anti-mouse IgG1. In separate experiments, HeLa cells were transiently transfected with TacNR2A and GFP-Rab9. Cells were incubated with Tac polyclonal antibody on ice for $1 \mathrm{hr}$ and then returned to conditioned media for 5,15 , or $30 \mathrm{~min}$ at $37^{\circ} \mathrm{C}$. The cells were fixed and permeabilized, and the immunoreactivity was visualized using Alexa 568-conjugated (red) anti-mouse secondary antibodies. For the statistical analysis, images from five different HeLa cells in three independent experiments were collected at a $63 \times$ objective with a Zeiss Axioplan 2 microscope and analyzed with the OpenLab colocalization module (Improvision). Values represent the mean \pm SEM of Pearson's correlation.
A
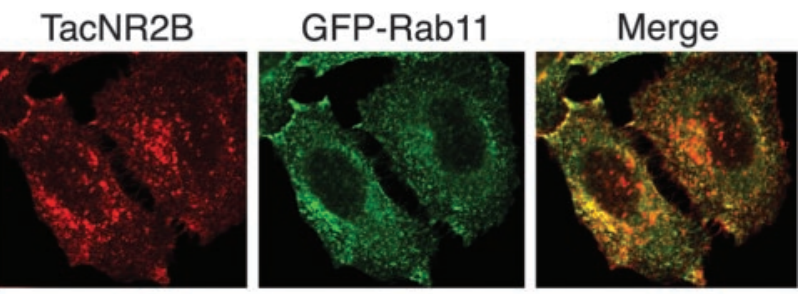

B

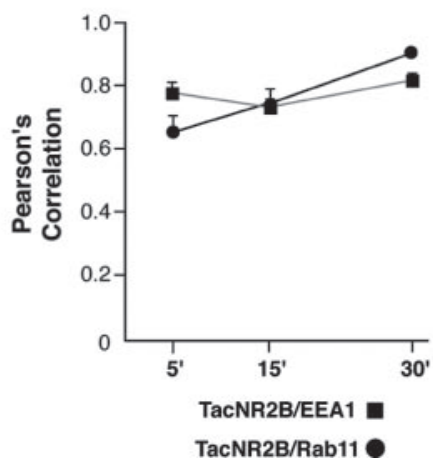

Figure 5. The NR2B C terminus regulates trafficking to recycling endosomes. $A$, HeLa cells were transiently transfected with TacNR2B and GFP-Rab11. Cells were incubated with Tac polyclonal antibody on ice for $1 \mathrm{hr}$ and then returned to conditioned media for $30 \mathrm{~min}$ at $37^{\circ} \mathrm{C}$. The cells were fixed and permeabilized, and the immunoreactivity of internalized TacNR2B was visualized using Alexa 568-conjugated (red) anti-mouse. GFP-Rab11 is visualized as green, and the merged image shows the level of colocalization of internalized TacNR2B with Rab11. B, Analysis of the amount of colocalization between TacNR2B and EEA1 or GFP-Rab11 was performed as described in the legend to Figure $4 E$. For the statistical analysis, images from five different HeLa cells in three independent experiments were collected at a $63 \times$ objective with a Zeiss Axioplan 2 microscope and analyzed with the OpenLab colocalization module (Improvision). Values represent the mean \pm SEM of Pearson's correlation.

ulates sorting from early endosomes into recycling endosomes. We then quantitated the colocalization of TacNR2B with the early endosomal marker EEA1 and the recycling endosomal marker GFP-Rab11 over time (5, 15, and $30 \mathrm{~min}$ ) (Fig. 5B), demonstrating that TacNR2B strongly colocalized with EEA1 at 5 min and that colocalization with Rab11 increased over time. The relatively high colocalization of TacNR2B with both EEA1 and GFP-Rab11 at all time points is consistent with continual cycling through this endocytic pathway.

\section{Developmental regulation of NR2A and NR2B trafficking} in neurons

Having characterized the trafficking of NR2A and NR2B chimeras in HeLa cells, we set out to determine whether NMDA receptors in neurons also displayed differential endocytosis dependent on subunit composition. We expressed full-length NR2A or NR2B proteins containing an extracellular FLAG epitope in hippocampal neurons at different stages of development. The use of the FLAG epitope tag allows us to specifically label surfaceexpressed NR2A or NR2B proteins on living cells. We transfected hippocampal neurons at 10 DIV with FLAG-NR2A or FLAGNR2B. At 12 DIV, we labeled the surface-expressed receptors with anti-FLAG antibody and returned the cells to $37^{\circ} \mathrm{C}$ for 15 min to allow internalization. The cells were then fixed and stained with Alexa 568 (red)-conjugated secondary antibody to label the surface population. The stained cells were then permeabilized, and Alexa 488 (green)-conjugated secondary antibody was added to specifically label the internalized population. Both NR2A and NR2B internalized in these neurons (Fig. 6A); however, the in- 
A
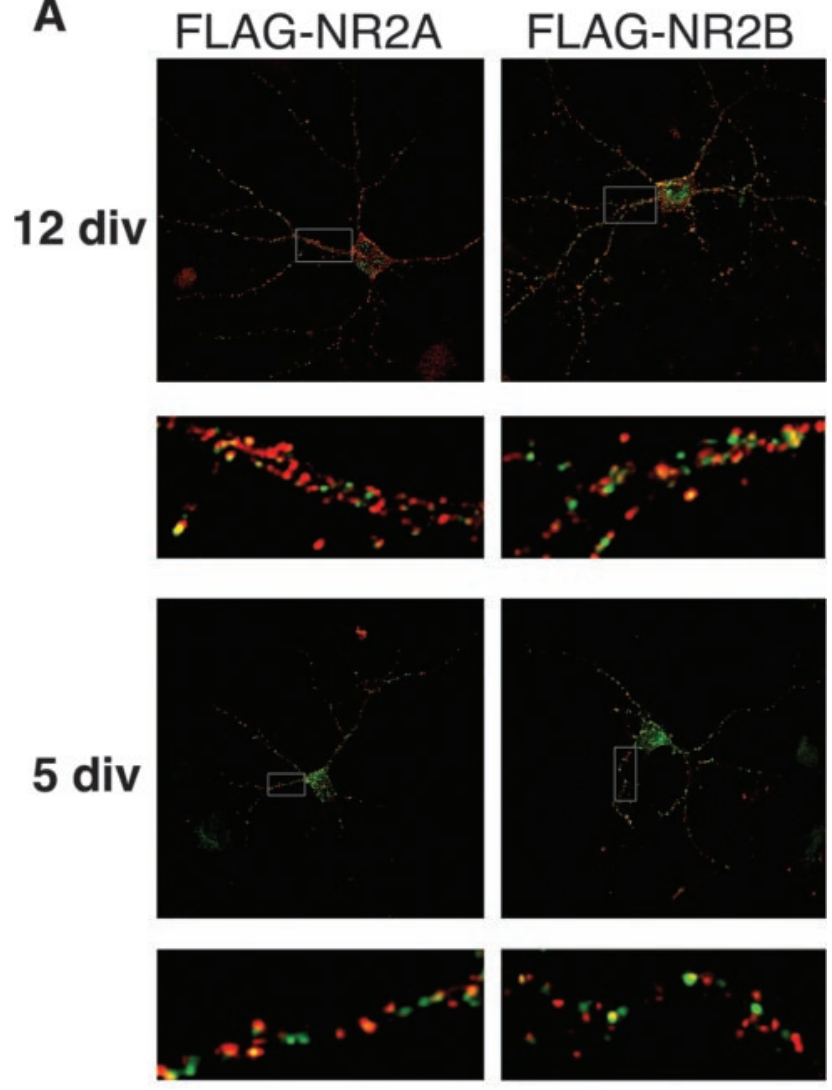

B

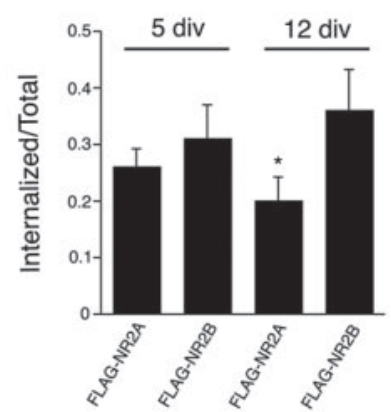

Figure 6. Differential internalization of NR2A and NR2B in hippocampal neurons during development. $A$, Hippocampal neurons (3 or 10 DIV) were transfected with FLAG-NR2B or FLAG-NR2A using the calcium phosphate coprecipitation method. After $2 \mathrm{~d}$ (at 5 or $12 \mathrm{DIV}$ ), the cells were incubated with FLAG polyclonal antibody for $45 \mathrm{~min}$ at room temperature. The antibody was removed, and the cells were returned to conditioned media for 15 min at $37^{\circ} \mathrm{C}$ to allow internalization. Neurons were fixed and incubated with Alexa 568-conjugated (red) anti-rabbit secondary antibody to visualize the surface pool. Cells were then washed, permeabilized, and incubated with Alexa 488-conjugated (green) anti-rabbit secondary antibody to specifically visualize the internalized pool. Green fluorescence indicated internalization compared with the total (red plus green). Images were collected with $63 \times$ objectives on a Zeiss LSM. Series of optical sections were collected at intervals of $0.34 \mu \mathrm{m}$. Maximum projections are shown. $B$, Histogram of the amount of internalization of FLAG-NR2A or FLAG-NR2B at 5 or 12 DIV. Series of optical sections were collected at intervals of $0.34 \mu \mathrm{m}$. Colocalization was measured with Volocity 2 software (Improvision). At 5 DIV, there is no significant difference between the amount of internalization of NR2A and NR2B, whereas at 12 DIV, there is a statistical difference between the two constructs. Data represent means \pm SEM; $n=9$; ${ }^{*} p<0.01$; Student's unpaired $t$ test.

ternalization of FLAG-NR2B was significantly greater than that of FLAG-NR2A (36 vs 20\%) (Fig. 6B).

It is possible that the NR2B subunit internalizes more efficiently than NR2A because of the extrasynaptic localization of
NR2B at sites separate from the scaffolding protein PSD-95. NR2A is only expressed later in development and is known to be synaptically localized, whereas NR2B is highly expressed at both synaptic and extrasynaptic sites (Li et al., 2002). Therefore, we evaluated the internalization of NR2A and NR2B at earlier developmental times, when the cells are less mature, when synapses are still developing, and when PSD-95 is known to be less abundant. We transfected hippocampal neurons at 3 DIV with FLAGNR2A or FLAG-NR2B and performed the immunofluorescence $2 \mathrm{~d}$ later as described above. Although FLAG-NR2A consistently internalized less well than FLAG-NR2B, the difference was not significant, and NR2A and NR2B displayed similar levels of endocytosis (26 vs $31 \%$ ) (Fig. 6). We subsequently analyzed whether the same mutations that inhibited the internalization of TacNR2A in HeLa cells had the same effect in hippocampal neurons. We transfected neurons at 3 DIV with FLAG-NR2A and FLAG-NR2A $\Delta 11$ L1320A but found no significant difference in the internalization rate between the two constructs (data not shown), suggesting that heteromeric complexes of NR2A in neurons may use this motif differently or less efficiently than the monomeric chimera in HeLa cells. Unfortunately, with the high expression of NR2B in primary neuronal cultures at all developmental stages, it is impossible for us to specifically evaluate subpopulations of heteromeric NR2A in neurons (for example, NR1-NR2A vs NR1-NR2B-NR2A).

\section{Differential sorting of NR2A and NR2B in hippocampal neurons}

Because both NR2A and NR2B internalize efficiently in hippocampal neurons at $5 \mathrm{DIV}$, we decided to use this developmental time to analyze the endocytic sorting and intracellular distribution of the internalized subunits. We coexpressed FLAGNR2A or FLAG-NR2B with various endosomal marker proteins (GFP-Rab5, GFP-Rab9, or GFP-Rab11) and analyzed colocalization at different time points. At $15 \mathrm{~min}$, FLAG-NR2A colocalizes well with the early endosome marker GFP-Rab5 (Fig. 7A) but to a lesser degree with the late endosome marker GFP-Rab9 (Fig. 7D) and the recycling endosome marker GFP-Rab11 (Fig. $7 D)$. At $30 \mathrm{~min}$, the colocalization of FLAG-NR2A with all three markers was similar (Fig. 7D). In contrast, at 15 min, FLAGNR2B colocalizes with all three markers (Fig. $8 A-C$ ), but most prominently with GFP-Rab5. At $30 \mathrm{~min}$, the amount of colocalization with the late endosome marker GFP-Rab9 decreased substantially (Fig. $8 B-D$ ), whereas colocalization of FLAG-NR2B with the recycling endosome marker GFP-Rab11 almost doubled (Fig. $8 C-D$ ), indicating the preferential sorting of NR2B through recycling endosomes. Quantitative analyses revealed that whereas only $25 \%$ of FLAG-NR2A colocalized with GFP-Rab11 after 30 min of internalization, almost 50\% of FLAG-NR2B colocalized with the recycling endosome marker under these conditions (Fig. $8 D$ ), demonstrating that NMDA receptors containing NR2B rather than NR2A have a clear preference for the recycling pathway. Together, these data extend our studies of NMDA receptor trafficking in heterologous cells to primary neuronal cultures and demonstrate distinct endocytic sorting motifs encoded within NR2A and NR2B.

\section{Discussion}

Recent studies have demonstrated that NMDA receptors are quite mobile, including intracellular pools of NMDA receptors (Standley et al., 2000; Scott et al., 2001; Xia et al., 2001; Washbourne et al., 2002), plasma membrane-expressed NMDA receptors, and even synaptic NMDA receptors (Rao and Craig, 1997; 


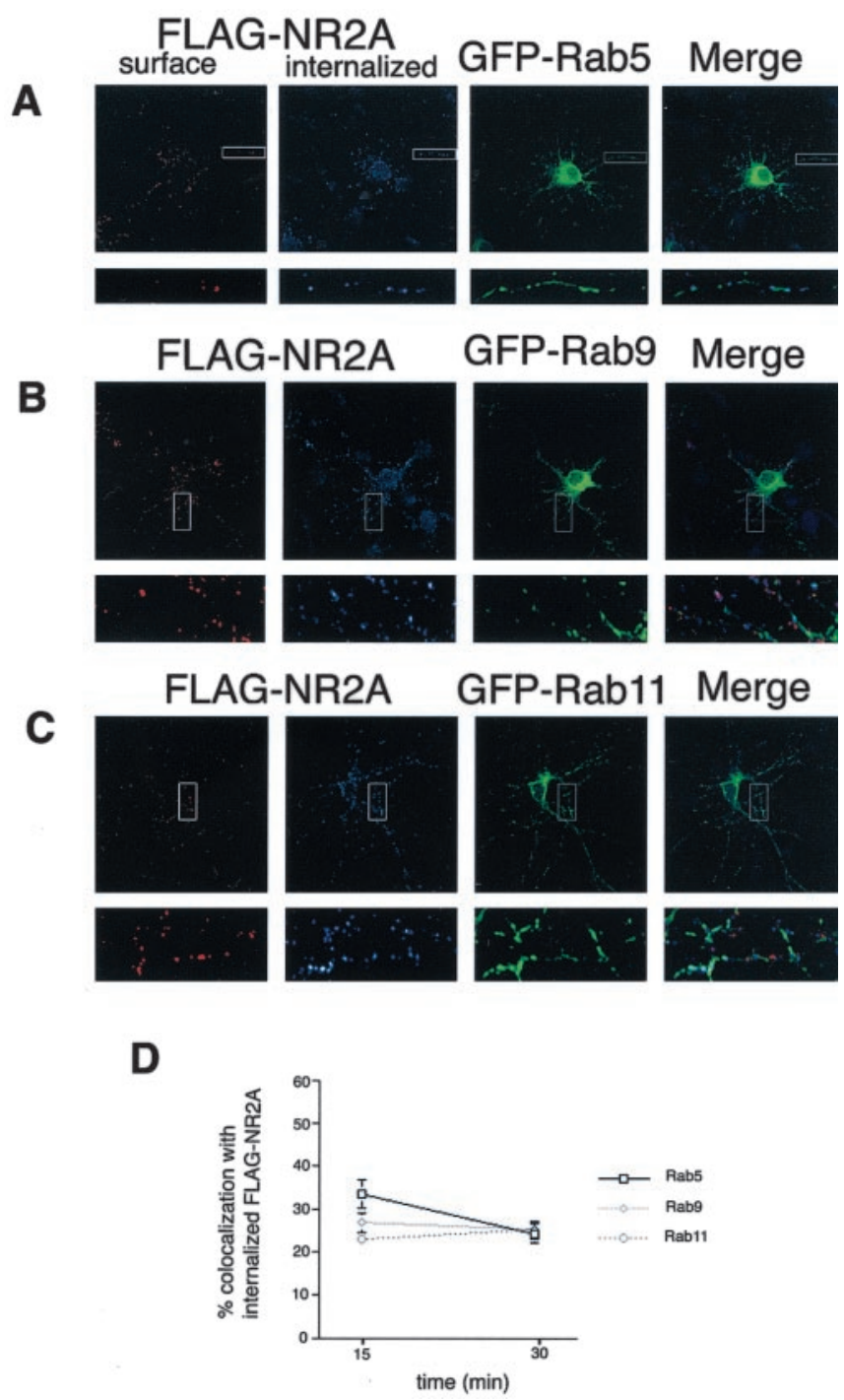

Figure 7. Internalized NR2A traffics through early, late, and recycling endosomes in hippocampal neurons. Hippocampal neurons (3 DIV) were transfected with FLAG-NR2A and GFPRab5 ( $A$ ), FLAG-NR2A and GFP-Rab9 ( $B$ ), or FLAG-NR2A and GFP-Rab11 ( $C$ using the calcium phosphate coprecipitation method. At 5 DIV, the cells were incubated with FLAG polyclonal antibody for 45 min at room temperature, antibody was removed, and the cells were returned to conditioned media for 15 or $30 \mathrm{~min}$ at $37^{\circ} \mathrm{C}$ to allow internalization. Neurons were then fixed and incubated with Alexa 568-conjugated (red) anti-rabbit secondary antibody to visualize the surface pool. Cells were then washed, permeabilized, and incubated with Cy5conjugated (blue) anti-rabbit secondary antibody to specifically visualize the internalized pool. Blue fluorescence indicated internalization compared with the total (red plus blue). Images were collected with $63 \times$ objectives on a Zeiss LSM. Maximum projections are shown. D, Statistical analysis of the amount of colocalization between FLAG-NR2A and GFP-Rab5, GFPRab9, or GFP-Rab11. Series of optical sections were collected at intervals of $0.34 \mu \mathrm{m}$. Images from three dendrites per neuron ( 3 neurons per experiment) were collected, and colocalization analysis was measured based on the data collected in three to five independent experiments with Volocity 2 software (Improvision). Values represent mean \pm SEM; $n=5$.

Rumbaugh and Vicini, 1999; Snyder et al., 2001; Grosshans et al., 2002; Prybylowski et al., 2002; Tovar and Westbrook, 2002). Furthermore, endocytosis of native NMDA receptors is developmentally regulated such that endocytosis is robust early in development but declines as neurons mature and synapses form (Roche et al., 2001). Interestingly, this change in endocytosis parallels changes in NMDA receptor subunit composition. In the forebrain early in development, NMDA receptors are thought to be NR1-NR2B heteromers that switch to heteromeric combina-
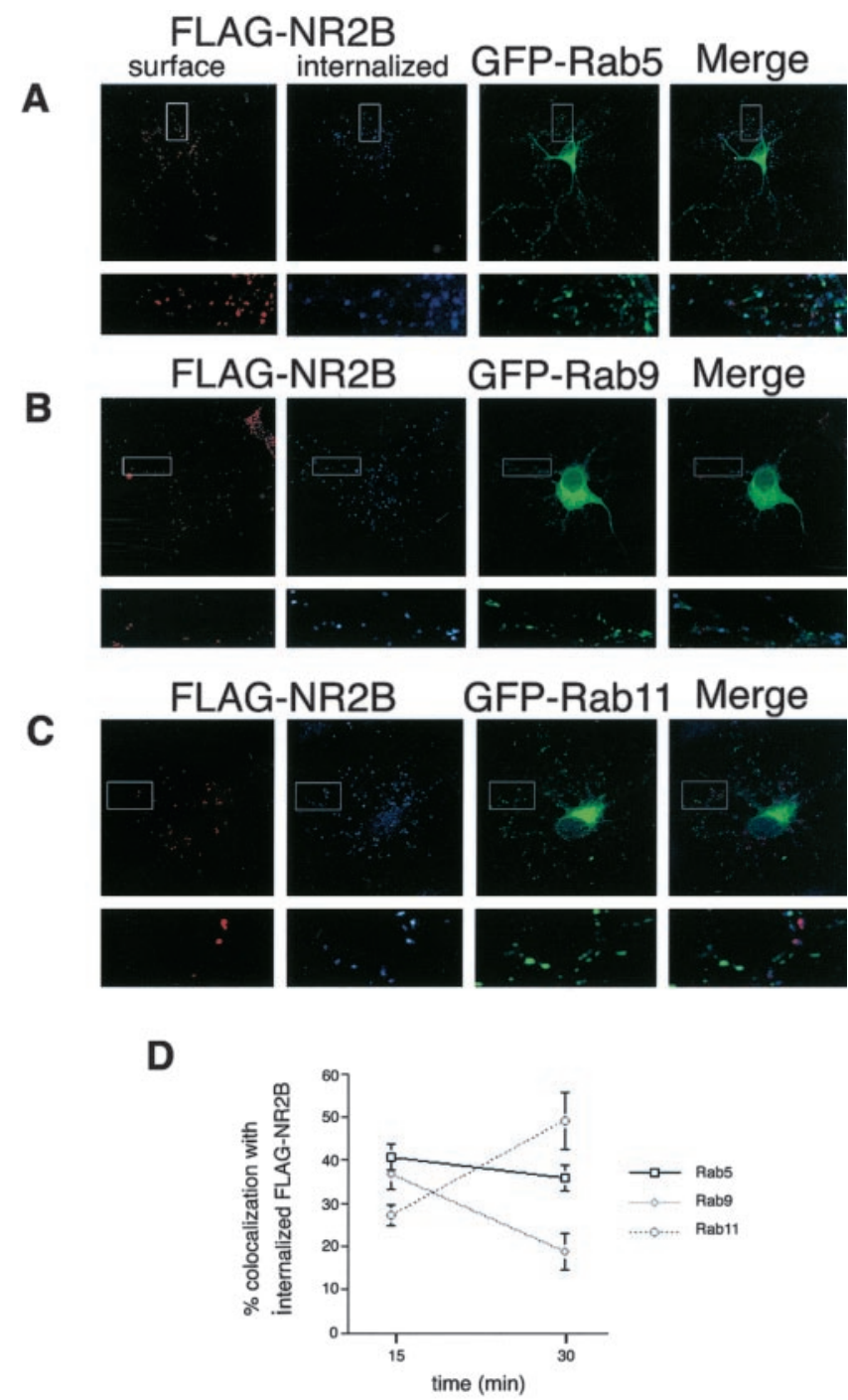

Figure 8. NR2B preferentially traffics through recycling endosomes in hippocampal neurons. Hippocampal neurons (3 DIV) were transfected with FLAG-NR2B and GFP-Rab5 $(A)$, FLAG-NR2B and GFP-Rab9 ( $B$ ), or FLAG-NR2B and GFP-Rab11 ( $C$ using the calcium phosphate coprecipitation method. At 5 DIV, the cells were incubated with FLAG polyclonal antibody for $45 \mathrm{~min}$ at room temperature, antibody was removed, and the cells were returned to conditioned media for 15 or $30 \mathrm{~min}$ at $37^{\circ} \mathrm{C}$ to allow internalization. Neurons were then fixed and incubated with Alexa 568-conjugated (red) anti-rabbit secondary antibody to visualize the surface pool. Cells were then washed, permeabilized, and incubated with Cy5-conjugated (blue) anti-rabbit secondary antibody to specifically visualize the internalized pool. Blue fluorescence indicated internalization compared with the total (red plus blue). Images were collected with $63 \times$ objectives on a Zeiss LSM. Series of optical sections were collected at intervals of $0.34 \mu \mathrm{m}$. Maximum projections are shown. D, Statistical analysis of the amount of colocalization between FLAG-NR2B and GFP-Rab5, GFP-Rab9, or GFP-Rab11. Series of optical sections were collected at intervals of $0.34 \mu \mathrm{m}$. Images from three dendrites per neuron ( 3 neurons per experiment) were collected, and colocalization analysis was measured based on the data collected in three to five independent experiments with Volocity 2 software (Improvision). Values represent mean $\pm \mathrm{SEM} ; n=5$.

tions of NR1, NR2A, and NR2B in the adult brain (Kew et al., 1998; Li et al., 1998; Rumbaugh and Vicini, 1999; Tovar and Westbrook, 1999). In the present study, we have probed the possibility that NR2A and NR2B differentially regulate plasma membrane expression and endocytic sorting of NMDA receptors. We found that NR2A and NR2B do indeed possess distinct endocytic motifs and sorting information within their distal $\mathrm{C}$ termini. In addition, we found that the NR2 subunit content of NMDA re- 
ceptors in hippocampal neurons regulates the amount of NMDA receptor endocytosis and determines the intracellular sorting of NMDA receptors after endocytosis. This demonstrates the important influence of subunit composition of NMDA receptors on trafficking, plasma membrane expression, and endocytosis.

NMDA receptors, like all integral membrane proteins expressed on the cell surface, must undergo regulated transport to the plasma membrane, where they can be selectively stabilized, undergo endocytosis-degradation, or undergo endocytosis followed by recycling to the plasma membrane. Protein trafficking through distinct intracellular pathways is tightly regulated primarily by specific proteinprotein interactions between cargo proteins and the trafficking machinery. One of our primary goals is to define motifs and molecular binding sites on NMDA receptors critical for regulating trafficking, surface expression, and endocytosis. Although in recent years there have been numerous studies published on glutamate receptor trafficking and endocytosis, it is interesting to note that AMPA receptors, the best-characterized subtype of glutamate receptor thus far, do not contain any of the strong consensus sequences known to play a role in clathrin-mediated endocytosis. In sharp contrast, the cytosolic domains of NMDA receptors have numerous consensus endocytic motifs. Thus it is likely that the mechanisms regulating the surface expression and synaptic targeting of AMPA receptors and NMDA receptors are distinct. In addition, NMDA receptors possess a variety of well characterized protein-binding motifs that regulate interactions with components of the PSD (Hung and Sheng, 2002). These proteins are also likely to mediate synaptic expression of NMDA receptors.

In this study, we have focused on elucidating differences between NR2A and NR2B that are important for NMDA receptor trafficking and developmental changes in surface expression. Both NR2 subunits need to associate with NR1 to get to the surface (McIlhinney et al., 1998). Similarly, Tac chimeras including the entire NR2A or NR2B C terminus are retained in the ER (data not shown) (Hawkins et al., 2004). Therefore, we focused on the distal portion of the NR2 C terminus for our studies and found that the NR2A C terminus, like that of NR2B, contains a dominant endocytic motif. Previous studies have characterized a tyrosine-based endocytic motif on NR2B, YEKL, which binds directly to the medium chain of AP-2 and can regulate endocytosis in heterologous cells and in neurons (Roche et al., 2001; Lavezzari et al., 2003). Although the tyrosine in NR2B (Tyr 1472) is conserved in NR2A (Tyr 1454), the surrounding residues are different, suggesting that the interaction of these proteins with clathrin-associated adaptors might also differ. We found that this tyrosine-based motif within the $\mathrm{C}$ terminus of NR2A did not regulate endocytosis; in this case, a dileucine motif (Leu 1319, Leu 1320) plays a critical role in NR2A-mediated internalization. Interestingly, as described previously for NR2B, we still found that coexpression with PSD-95 inhibited NR2A-mediated endocytosis. Thus for both NR2A and NR2B, binding of PSD-95 inhibits clathrin-mediated endocytosis.

Our evidence that endocytic motifs in the distal C termini of NMDA receptors were different in the individual NR2 subunits prompted us to perform binding assays of the NR2A and NR2B C termini with the medium chains of the various clathrin adaptor complexes $(\mu 1-\mu 4)$. Interestingly, we found that although both NR2A and NR2B C termini could directly interact with adaptor medium chains, the specificity varied. For example, the binding to $\mu 1$, the medium chain for AP-1 that regulates trafficking at the TGN, was similar for NR2A and NR2B, whereas the binding to $\mu 2$ was quite different. We found that NR2B binds very well to $\mu 2$ (Fig. 3) (Lavezzari et al., 2003); however, the NR2A C terminus displays almost no binding. This is consistent with the YEKL motif within NR2B being important in plasma membrane endocytosis, unlike the YKKM motif of NR2A. However, our findings still clearly demonstrate that the $\mathrm{C}$ termini of NR2A and NR2B both directly interact with medium chains of clathrin adaptor proteins and are both likely to interact with the clathrin machinery at various intracellular sorting steps. It is important to note that the preference of NR2A for $\mu 1$ versus $\mu 2$ is somewhat rare. It has even been proposed in the literature (Ohno et al., 1996; Bonifacino and Traub, 2003) that $\mu 2$ binding is almost a default interaction for any sequence that interacts with clathrin adaptor medium chains. Specificity for a particular medium chain is more common for $\mu 1$, for example. These data suggest that the lack of $\mu 2$ binding to the NR2A C terminus is of particular importance.

In addition to the distal $\mathrm{C}$ terminus of NR2A and NR2B that we have characterized in the present study, there is good evidence that a region adjacent to transmembrane domain 4 (TM4) of the NMDA receptor subunits is important for surface expression and functional regulation of NMDA receptors (Vissel et al., 2001). Interestingly, there is a conserved tyrosine in all NMDA receptor subunits adjacent to TM4 that appears to be sensitive to phosphorylation, and mutation of this residue of NR2A influences channel function and rundown in rat hippocampal pyramidal neurons and heterologous cells (Vissel et al., 2001). It is possible that these conserved tyrosines form a ring near the plasma membrane of NMDA receptors and may regulate plasma membrane expression or trafficking through intracellular pathways. One of the challenges for the future will be to determine how these motifs in different regions of NMDA receptors work together to mediate trafficking, surface expression, and/or endocytosis of endogenous NMDA receptors.

Our evidence indicates that NR2B undergoes more robust endocytosis than NR2A. This could be attributable to several factors. Many groups have shown that NR2A is highly synaptic, whereas NR2B is expressed at both synaptic and extrasynaptic sites (Stocca and Vicini, 1998; Tovar and Westbrook, 1999; Li et al., 2002). This is consistent with NR2A being strictly colocalized with components of the PSD that are likely to stabilize synaptic receptors, most notably PSD-95. Additional support for this hypothesis is that NR2A expression and PSD-95 expression are coordinately increased during development. Conversely, NR2B is expressed not only early in development during synaptogenesis but also in mature neurons at extrasynaptic sites. We also demonstrated that the NR2A and NR2B subunits direct differential sorting into distinct intracellular pathways after endocytosis. The NR2A and NR2B C termini both initially regulate internalization into early endosomes; however, over time, these proteins diverge and NR2A traffics to late endosomes, whereas NR2B preferentially sorts into recycling endosomes. These data fit well with the described higher level of NMDA receptor endocytosis early in development (Roche et al., 2001), with NR2B preferring the recycling pathway (Figs. 5, 8), and with high expression of NR2B at extrasynaptic sites in mature neurons. Our finding that NR2B is more likely to recycle seems quite logical, because NR2B is highly expressed early in development, when endocytosis is more robust. Given the relatively long half-life of both surface and total pools of glutamate receptors (Hall and Soderling, 1997; Mammen et al., 1997; Huh and Wenthold, 1999) and the fact that NR2B endocytosis occurs at a rapid rate (31-36\% of NR2B-containing receptors in 15 min) (Fig. 6), if recycling of NR2B were not an ongoing process, the neuron would rapidly deplete the surface pool of NMDA receptors.

Our findings support a model of NMDA receptor endocytosis that is more robust early in development and at extrasynaptic sites, conditions in which PSD-95 expression is limited and is therefore 
not available to stabilize plasma membrane-expressed NMDA. When NMDA receptors are expressed at the plasma membrane at nonsynaptic sites, their $\mathrm{C}$ termini are free to bind to the endocytic machinery and the receptors undergo endocytosis. In support of our model, other studies of NMDA receptor function at synaptic versus extrasynaptic sites demonstrate a role for endocytosis regulated by tyrosine phosphorylation of NMDA receptor and subsequent regulation of rundown (Wang et al., 1996; Li et al., 2002). These data fit well with our findings on a differential contribution of NR2A and NR2B to NMDA receptor endocytosis and the differential sorting of distinct NMDA receptor complexes. Our evidence of the direct interaction of NR2A and NR2B with clathrin adaptor medium chains is also supported by recent findings that NMDA receptors coimmunoprecipitate with adaptor complexes (Nong et al., 2003), demonstrating the importance of clathrin-mediated endocytosis and NMDA receptor regulation.

Our studies elucidated critical differences between the NR2 subunits that are expressed in the hippocampus and cortex. Using a variety of approaches, we demonstrate that the NR2A and NR2B subunits have dominant endocytic motifs that regulate sorting into distinct intracellular pathways. These differences are also seen when NR2A and NR2B are expressed and their endocytosis is monitored in primary neuronal culture. These findings provide a framework for understanding developmental changes in NMDA receptor subunit expression and how it regulates NMDA receptor trafficking and synaptic expression.

\section{References}

Aguilar RC, Boehm M, Gorshkova I, Crouch RJ, Tomita K, Saito T, Ohno H, Bonifacino JS (2001) Signal-binding specificity of the $\mu 4$ subunit of the adaptor protein complex AP-4. J Biol Chem 276:13145-13152.

Bonifacino JS, Traub LM (2003) Signals for sorting of transmembrane proteins to endosomes and lysosomes. Annu Rev Biochem 72:395-447.

Grosshans DR, Clayton DA, Coultrap SJ, Browning MD (2002) LTP leads to rapid surface expression of NMDA but not AMPA receptors in adult rat CA1. Nat Neurosci 5:27-33.

Hall RA, Soderling TR (1997) Quantitation of AMPA receptor surface expression in cultured hippocampal neurons. Neuroscience 78:361-371.

Hawkins LM, Prybylowski K, Chang K, Moussan C, Stephenson FA, Wenthold RJ (2004) Export from the endoplasmic reticulum of assembled NMDA receptors is controlled by a motif in the $\mathrm{C}$ terminus of the NR2 subunit. J Biol Chem, in press.

Huh KH, Wenthold RJ (1999) Turnover analysis of glutamate receptors identifies a rapidly degraded pool of the $N$-methyl-D-aspartate receptor subunit, NR1, in cultured cerebellar granule cells. J Biol Chem 274:151-157.

Hung AY, Sheng M (2002) PDZ domains: structural modules for protein complex assembly. J Biol Chem 277:5699-5702.

Kew JN, Richards JG, Mutel V, Kemp JA (1998) Developmental changes in NMDA receptor glycine affinity and ifenprodil sensitivity reveal three distinct populations of NMDA receptors in individual rat cortical neurons. J Neurosci 18:1935-1943.

Kim E, Niethammer M, Rothschild A, Jan YN, Sheng M (1995) Clustering of Shaker-type $\mathrm{K}+$ channels by interaction with a family of membraneassociated guanylate kinases. Nature 378:85-88.

Kornau HC, Schenker LT, Kennedy MB, Seeburg PH (1995) Domain interaction between NMDA receptor subunits and the postsynaptic density protein PSD-95. Science 269:1737-1740.

Lavezzari G, McCallum J, Lee R, Roche KW (2003) Differential binding of the AP-2 adaptor complex and PSD-95 to the C-terminus of the NMDA receptor subunit NR2B regulates surface expression. Neuropharmacology 45:729-737.

Li B, Chen N, Luo T, Otsu Y, Murphy TH, Raymond LA (2002) Differential regulation of synaptic and extra-synaptic NMDA receptors. Nat Neurosci 5:833-834.

Li JH, Wang YH, Wolfe BB, Krueger KE, Corsi L, Stocca G, Vicini S (1998) Developmental changes in localization of NMDA receptor subunits in primary cultures of cortical neurons. Eur J Neurosci 10:1704-1715.
Luscher C, Xia H, Beattie EC, Carroll RC, von Zastrow M, Malenka RC, Nicoll RA (1999) Role of AMPA receptor cycling in synaptic transmission and plasticity. Neuron 24:649-658.

Malinow R, Malenka RC (2002) AMPA receptor trafficking and synaptic plasticity. Annu Rev Neurosci 25:103-126.

Mammen AL, Huganir RL, O’Brien RJ (1997) Redistribution and stabilization of cell surface glutamate receptors during synapse formation. J Neurosci 17:7351-7358.

McIlhinney RA, Le Bourdelles B, Molnar E, Tricaud N, Streit P, Whiting PJ (1998) Assembly intracellular targeting and cell surface expression of the human $\mathrm{N}$-methyl-D-aspartate receptor subunits NR1a and NR2A in transfected cells. Neuropharmacology 37:1355-1367.

Nong Y, Huang YQ, Ju W, Kalia LV, Ahmadian G, Wang YT, Salter MW (2003) Glycine binding primes NMDA receptor internalization. Nature 422:302-307.

Ohno H, Stewart J, Fournier MC, Bosshart H, Rhee I, Miyatake S, Saito T, Gallusser A, Kirchhausen T, Bonifacino JS (1995) Interaction of tyrosine-based sorting signals with clathrin-associated proteins. Science 269:1872-1875.

Ohno H, Fournier MC, Poy G, Bonifacino JS (1996) Structural determinants of interaction of tyrosine-based sorting signals with the adaptor medium chains. J Biol Chem 271:29009-29015.

Prybylowski K, Fu Z, Losi G, Hawkins LM, Luo J, Chang K, Wenthold RJ, Vicini S (2002) Relationship between availability of NMDA receptor subunits and their expression at the synapse. J Neurosci 22:8902-8910.

Rao A, Craig AM (1997) Activity regulates the synaptic localization of the NMDA receptor in hippocampal neurons. Neuron 19:801-812.

Roche KW, Huganir RL (1995) Synaptic expression of the high-affinity kainate receptor subunit KA2 in hippocampal cultures. Neuroscience 69:383-393.

Roche KW, Standley S, McCallum J, Dune Ly C, Ehlers MD, Wenthold RJ (2001) Molecular determinants of NMDA receptor internalization. Nat Neurosci 4:794-802.

Rumbaugh G, Vicini S (1999) Distinct synaptic and extrasynaptic NMDA receptors in developing cerebellar granule neurons. J Neurosci 19:10603-10610.

Scott DB, Blanpied TA, Swanson GT, Zhang C, Ehlers MD (2001) An NMDA receptor ER retention signal regulated by phosphorylation and alternative splicing. J Neurosci 21:3063-3072.

Sheng M (2001) Molecular organization of the postsynaptic specialization. Proc Natl Acad Sci USA 98:7058-7061.

Snyder EM, Philpot BD, Huber KM, Dong X, Fallon JR, Bear MF (2001) Internalization of ionotropic glutamate receptors in response to mGluR activation. Nat Neurosci 4:1079-1085.

Standley S, Roche KW, McCallum J, Sans N, Wenthold RJ (2000) PDZ domain suppression of an ER retention signal in NMDA receptor NR1 splice variants. Neuron 28:887-898.

Stocca G, Vicini S (1998) Increased contribution of NR2A subunit to synaptic NMDA receptors in developing rat cortical neurons. J Physiol (Lond) 507:13-24.

Tovar KR, Westbrook GL (1999) The incorporation of NMDA receptors with a distinct subunit composition at nascent hippocampal synapses in vitro. J Neurosci 19:4180-4188.

Tovar KR, Westbrook GL (2002) Mobile NMDA receptors at hippocampal synapses. Neuron 34:255-264.

Vicini S, Wang JF, Li JH, Zhu WJ, Wang YH, Luo JH, Wolfe BB, Grayson DR (1998) Functional and pharmacological differences between recombinant $N$-methyl-D-aspartate receptors. J Neurophysiol 79:555-566.

Vissel B, Krupp JJ, Heinemann SF, Westbrook GL (2001) A use-dependent tyrosine dephosphorylation of NMDA receptors is independent of ion flux. Nat Neurosci 4:587-596.

Wang YT, Yu XM, Salter MW (1996) $\mathrm{Ca}^{2+}$-independent reduction of $N$-methyl-D-aspartate channel activity by protein tyrosine phosphatase. Proc Natl Acad Sci USA 93:1721-1725.

Washbourne P, Bennett JE, McAllister AK (2002) Rapid recruitment of NMDA receptor transport packets to nascent synapses. Nat Neurosci 5:751-759.

Wenthold RJ, Prybylowski K, Standley S, Sans N, Petralia RS (2003) Trafficking of NMDA receptors. Annu Rev Pharmacol Toxicol 43:335-358.

Xia H, Hornby ZD, Malenka RC (2001) An ER retention signal explains differences in surface expression of NMDA and AMPA receptor subunits. Neuropharmacology 41:714-723. 